\title{
ASCO 2014: news, hopes, hypes?
}

\author{
Wolfgang Eisterer
}

Received: 6 November 2014 / Accepted: 17 November 2014 / Published online: 28 November 2014 (C) Springer-Verlag Wien 2014

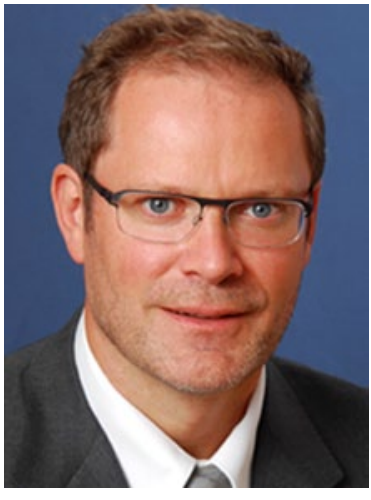

Wolfgang Eisterer
This year's American Society of Clinical Oncology (ASCO) saw some major improvements in the treatment of patients with solid tumors which, however, did not lead to a change in current clinical practice; at least not in the treatment of those solid tumors which will be reported within this summary. Surprisingly not all the therapeutic progress stems from targeted therapy but also from longknown substances like aromatase inhibitors. In total, more than 5000 abstracts have been presented at ASCO 2014. Within this review the most important abstracts covering head and neck tumors, breast cancer, melanoma, lung cancer, and colorectal cancer will be presented and put into context regarding actual clinical practice.

In the field of breast cancer, we have learned that combined ovarian function suppression (OFS) plus exemestane might be superior to OFS plus tamoxifen in premenopausal early breast cancer patients [1] with regard to disease-free survival (DFS). Whether OFS plus exemestane already represents a new standard is too early to comment on since no data on the comparison of OFS plus exemestane versus tamoxifen alone, which represents the actual standrad therapy, have been presented.

Dual Her2-inhibition with trastuzumab and lapatinib given either concomitantly or sequentially failed to demonstrate an improvement in DFS or overall survival (OS) in the adjuvant treatment of early breast cancer [2]. Results of another study on dual Her2-blockade with trastuzumab and pertuzumab are not available yet; therefore, it is not possible to draw firm conclusions on the role of dual Her2-receptor-blocking in the adjuvant setting. Trastuzumab given for 1 year remains the standard of care in this indication as is outlined by Rupert Bartsch and Elisabeth Bergen in their excellent overview.

\section{W. Eisterer $(\bowtie)$}

Department of Internal Medicine V, Medical University Innsbruck, Anichstraße 35, 6020 Innsbruck, Austria

e-mail: wolfgang.eisterer@i-med.ac.at
In the treatment of locally advanced head and neck tumors concomitant chemoradiotherapy is considered as standard of care. Owing to the fact that systemic relapse occurs frequently during follow-up in these patients strategies that include systemic therapy have been developed. Special interest has focused on the concept of sequential therapy meaning induction chemotherapy before chemoradiotherapy. Numerous phase III studies have failed to show an improvement in survival for induction chemotherapy [3-5]. An Italian phase III study was able to demonstrate improved survival for patients with locally advanced squamous carcinoma of the head and neck using induction chemotherapy as an add-on to definitive local chemoradiotherapy or radiotherapy plus cetuximab [6]. The definitive role of sequential therapy remains open as is concluded by Georg Pall in his thorough review.

During the past 50 years, seven drugs have been approved by the US Food and Drug Administration (FDA) for the management of melanoma. Three drugs (dacarbazine, interferon alpha-2b, and interleukin-2) were approved approximately 20 years ago; most remarkably, four drugs (vemurafenib, dabrafenib, trametinib, and ipilimumab) were all FDA approved within the past 3 years. The discovery of the pathogenic activating BRAF V600E mutation in cutaneous melanoma allowed for an unprecedented pace of drug development.

At ASCO 2014, researchers reported results from a large clinical trial testing the dabrafenib/trametinib combination in volunteer patients. The trial, called COMBI-d [7], compared the new treatment to dabrafenib alone and confirmed that the combination treatment performs better: $93 \%$ of the patients who took dabrafenib/trametinib were alive after 6 months, compared with $85 \%$ of the patients who took only dabrafenib.

Immunotherapy treatments boost a patient's own immune system to fight cancer. There has been particular interest in immunotherapy drugs called "immune check- 
point inhibitors," which release brakes on immune system cells, freeing them to attack tumors. Georg Weinlich reports on very promising results when various of these immune checkpoint inhibitors are combined together as well as on new compounds that enrich the therapeutic armamentarium.

In lung cancer steady progress has been made within the last years with the discovery of genetic driver mutations which can be targeted with specific compounds such as tyrosine kinase inhibitors (TKI). TKI are already the mainstay of first-line therapy in non-small cell lung cancer (NSCLC) with mutations of the epidermal growth factor receptor (EGFR) pathway. A pooled analysis [8] of two randomized phase III trials comparing the secondgeneration EGFR- (and panHer-) TKI afatinib to platinum-duplets was presented, including only patients with the classical activating mutations Del19 and L858R. Compared with chemotherapy afatinib led to a significant OS prolongation (HR 0.81).

The treatment algorithms for squamous-cell NSCLC have been unchanged since the approval of erlotinib for second/third-line treatment in 2005. Necitumumab, a fully human IgG1 anti-EGFR-antibody has now been tested as an add-on to a standard platinum-based doublet in the first-line treatment of metastatic squamous NSCLC (SQUIRE-trial) [9] and resulted in a statistically significant benefit in OS (HR 0.84; $p=0.012$; median OS 9.9 versus 11.5 months) as is reported by Ferdinand Ploner.

At last but not least, two studies have been presented on the question which antibody should be paired with chemotherapy for the first-line treatment of metastatic colorectal cancer. While in the CALGB 80405 trial [10] no significant survival difference could be observed between chemotherapy plus cetuximab or bevacizumab another phase III trial, FIRE 3 [11], showed improved OS (secondary endpoint) when first-line therapy was started with cetuximab plus chemotherapy instead of chemotherapy plus bevacizumab. Wolfgang Eisterer will report on these results and further important trials within the field of colorectal cancer.

I hope that $M E M O$ readers can gain insights and valuable information for daily clinical practice. The steps we take even with targeted therapies are not always big steps but rather small steps within a complex network of tumor biology.

\section{Conflict of interest}

The author has no conflicts of interest to disclose.

\section{References}

1. Pagani O, Regan MM, Walley B, et al. Randomized comparison of adjuvant aromatase inhibitor (AI) exemestane (E) plus ovarian function suppression (OFS) vs tamoxifen (T) plus OFS in premenopausal women with hormone receptor-positive (HR+) early breast cancer (BC): joint analysis of IBCSG TEXT and SOFT trials. J Clin Oncol. 2014;32:5s (suppl; abstr LBA1).
2. Piccart-Gebhart MJ, Holmes AP, Baselga J, et al. First results from the phase III ALTTO trial (BIG 2-06; NCCTG [Alliance] N063D) comparing one year of anti-HER2 therapy with lapatinib alone, trastuzumab alone, their sequence, or their combination in the adjuvant treatment of HER2positive early breast cancer. J Clin Oncol. 2014;32(Suppl. 18):LBA4.

3. Haddad R, O'Neill AO, Rabinowits G, et al. Induction chemotherapy followed by concurrent chemoradiotherapy (sequential chemoradiotherapy) versus concurrent chemoradiotherapy alone in locally advanced head and neck cancer (PARADIGM): a randomized phase 3 trial. Lancet Oncol. 2013;14(3):257-64.

4. Hitt R, Grau JJ, Lopez-Pousa A, et al. A randomized phase III trial comparing induction chemotherapy followed by chemoradiotherapy versus chemoradiotherapy alone as treatment of unresectable head and neck cancer. Ann Oncol. 2014;25:216-25.

5. Cohen EEW, Karrison TG, Kocherginsky M, et al. Phase III randomized trial of induction chemotherapy in patients with N2 or N3 locally advanced head and neck cancer. J Clin Oncol. 2014;32:2735-43.

6. Ghi MG, Paccagnella A, Ferrari D, et al. Concomitant chemoradiation (CRT) or cetuximab/RT (CET/RT) versus induction Docetaxel/Cisplatin/5-Flourouracil (TPF) followed by CRT or CET/RT in patients with locally advanced squamous cell carcinoma of the head and neck (LASCCHN). J Clin Oncol. 2014;32:5s (abstract 6004).

7. Long GV, Stroyakovsky DL, Gogas H, et al. COMBI-d: a randomized, double-blinded, phase III study comparing the combination of dabrafenib and trametinib to dabrafenib and trametinib placebo as first-line therapy in patients (pts) with unresectable or metastatic BRAFV600E/K mutationpositive cutaneous melanoma. J Clin Oncol. 2014;32:5s (abstr 9011).

8. Yang JC, Sequist LV, Schuler M, et al. Overall survival (OS) in patients (pts) with advanced non-small cell lung cancer (NSCLC) harboring common (Del19/L858R) epidermal growth factor receptor mutations (EGFRmut): pooled analysis of two large open-label phase III studies (LUX-Lung 3 and LUX-Lung 6) comparing afatinib with chemotherapy (CT). J Clin Oncol. 2014;32;5s (suppl; abstr 8004).

9. Thatcher N, Hirsch FR, Szczesna A, et al. A randomized, multicenter, open-label, phase III study of gemcitabine-cisplatin (GC) chemotherapy plus necitumumab (IMC-11F8/ LY3012211) versus GC alone in the first-line treatment of patients (Pts) with stage IV squamous non-small cell lung cancer (sq-NSCLC). J Clin Oncol. 2014;32:5s (suppl; abstr 8008).

10. Venook A, Niedzwiecki D, Lenz HJ, et al. CALGB/SWOG 80405: Phase III trial of irinotecan/5-FU/leucovorin (FOLFIRI) or oxaliplatin/5-FU/leucovorin (mFOLFOX6) with bevacizumab (BV) or cetuximab (CET) for patients (pts) with KRAS wild-type (wt) untreated metastatic adenocarcinoma of the colon or rectum (MCRC). J Clin Oncol. 2014;32:5s (suppl; abstr LBA3).

11. Stintzing S, Jung A, Rossius L, et al. Analysis of KRAS/NRAS and BRAF mutations in FIRE-3: a randomized phase III study of FOLFIRI plus cetuximab or bevacizumab as firstline treatment for wild-type (WT) KRAS (exon 2) metastatic colorectal cancer (mCRC) patients. Proc ESMO. 2013, Abstract LBA17. 